\title{
On the origins of tumour subtypes
}

DOI:

10.1038/nrc2234
Clinical subtypes of epithelial tumours arising in a single organ are quite diverse. Although many of these differences have been attributed to specific genetic and epigenetic alterations, it has been less clear whether the cell of origin for each tumour subtype has a role. Tan Ince, Robert Weinberg and colleagues now demonstrate that different mammary epithelial cells of origin can give rise to tumours with distinctly different phenotypes.

Human mammary epithelial cells (HMECs) are traditionally propagated in vitro using MEGM culture medium. Transformed HMECs give rise to tumours resembling a rare subtype of human breast cancer (squamous cell carcinomas) when transplanted into mice. Ince, Weinberg and colleagues proposed that alternative culture conditions might allow the outgrowth of different normal epithelial cell types, and that transformation of these different cell types might produce different tumour types.

The authors first developed a new culture medium, termed WIT. They collected normal human breast tissue from reduction mammoplasties and cultured the cells in either WIT or MEGM medium. Two distinct populations of cells, termed BPECs (breast primary epithelial cells) or HMECs, respectively, grew in these different conditions. Analysis of gene-expression profiles of the two cell populations indicated that they have distinct differentiation states - BPECs are more myoepithelial than HMECs.

Because BPECs and HMECs were derived from the same donor, the authors were able to use them to test whether differences between these cells could affect the phenotypes of tumours derived from them. BPECs and HMECs were transformed in parallel by retroviral vectors encoding telomerase (TERT), SV40 early proteins and HRAS (the cells were termed BPLER and HMLER, respectively). Tumours formed from HMLER cells injected into the mammary fat pad of immunocompromised mice resembled squamous cell carcinomas, as reported previously. However, BPLER cells formed tumours similar to human breast adenocarcinomas, and were more similar to these very common human tumours than most breast tumour xenograft models. Interestingly, more than $70 \%$ of mice with BPLER tumours, but none of the mice with HMLER tumours, developed lung micrometastases after 10 weeks. In addition, significantly fewer BPLER cells than HMLER cells were required to initiate tumours ( 100 cells versus $2-3 \times 10^{6}$ cells). Comparison of the gene-expression profiles of BPLER and HMLER cells indicated that the cells were more similar to their non-transformed counterparts than to each other; there were significant differences in the expression of 3,213 genes between the two tumorigenic cell types, indicating that the same transformation conditions yielded gene-expression changes that were dependent on the nature of the precursor cell.

Ince, Weinberg and colleagues have shown that the in vitro cell-oforigin seems to have a significant role in determining the phenotype of the tumour that that cell gives rise to in a mouse fat pad, but further study will be required to determine whether distinct cells of origin also influence the observed differences among various human breast cancer subtypes. Sarah Seton-Rogers

ORIGINAL RESEARCH PAPER Ince, T. A et al. Transformation of different human breast epithelial cell types leads to distinct tumour phenotypes. Cancer Cell 12, 160-170 (2007)

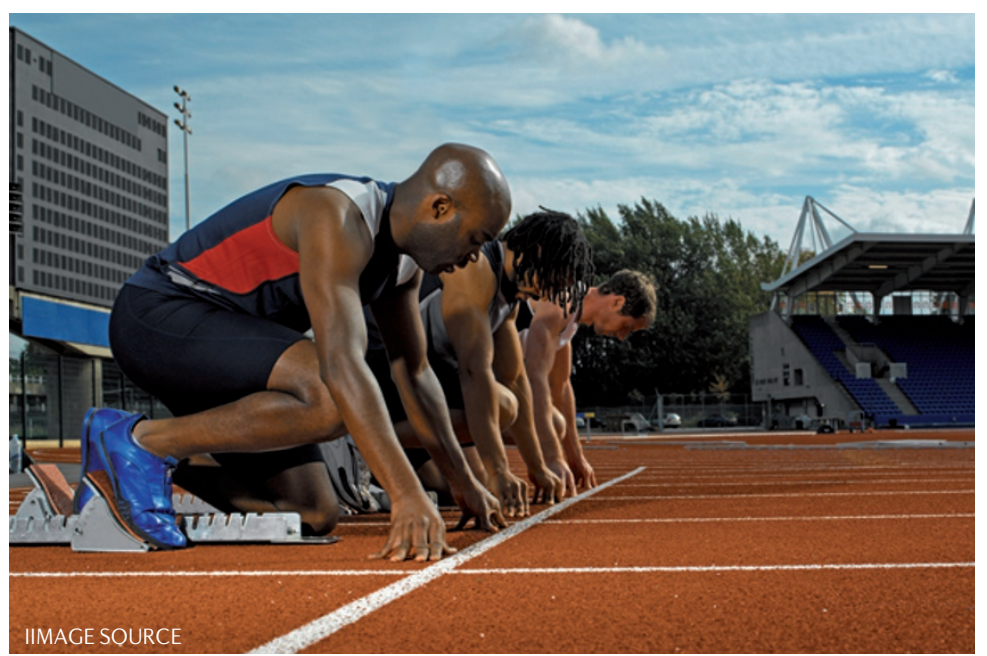

